\title{
Contact and Intersection Representations
}

\author{
Hubert de Fraysseix and Patrice Ossona de Mendez \\ UMR 8557, CNRS, Paris, France
}

\begin{abstract}
A necessary and sufficient condition is given for a connected bipartite graph to be the incidence graph of a family of segments and points. We deduce that any 4-connected 3-colorable plane graph is the contact graph of a family of segments and that any 4-colored planar graph without an induced $C_{4}$ using 4 colors is the intersection graph of a family of straight line segments.
\end{abstract}

To Chantal. Her life crossed mine on a too short path.

\section{Introduction}

Touchings and crossings of arcs in the plane have been the subject of lively interest, giving rise to astonishingly complex problems, albeit easy to state. As an example, the Gauss problem on the characterization of crossing sequences of self-intersecting closed curves [12], which has been fully solved only recently [6, 23]. The algebraic matroidal properties used to solve this problem further led to a characterization of bipartite circle graphs [3] and then to a characterization of general circle graphs à la Whitney [4].

Intersection graphs of arcs, the so-called string graphs, have been independently introduced by Sinden [27], Ehrlich, Even and Tarjan [11]. Their approach appeared to be quite complex $[15,17]$. The recognition problem has been proven to be NP-hard [16] and, more recently, NP-complete [21, 24].

The particular cases of intersection graphs of pseudo-segments and intersection graphs of segments [18] are of special interest, as shown by the following question by Scheinerman [25]: Is every planar graph the intersection graph of a set of segments in the plane?

This question is still open even for pseudo-segments, but some partial results have been obtained:

- the recognition problem of contact graphs of segments is NP-complete, even when restricted to planar graphs [14],

- bipartite planar graphs are contact graphs of a set of orthogonal segments $[9,13]$ (see also [1]),

- triangle-free planar graphs are necessarily contact graphs of a set of segments in three directions [2],

- 4-connected 3-colorable plane graphs are contact graphs of a set of pseudosegments [5],

- 4-colored plane graphs without $C_{4}$-separator using 4 colors are intersection graphs of a set of pseudo-segments [5] (see Fig.1 to 2). 

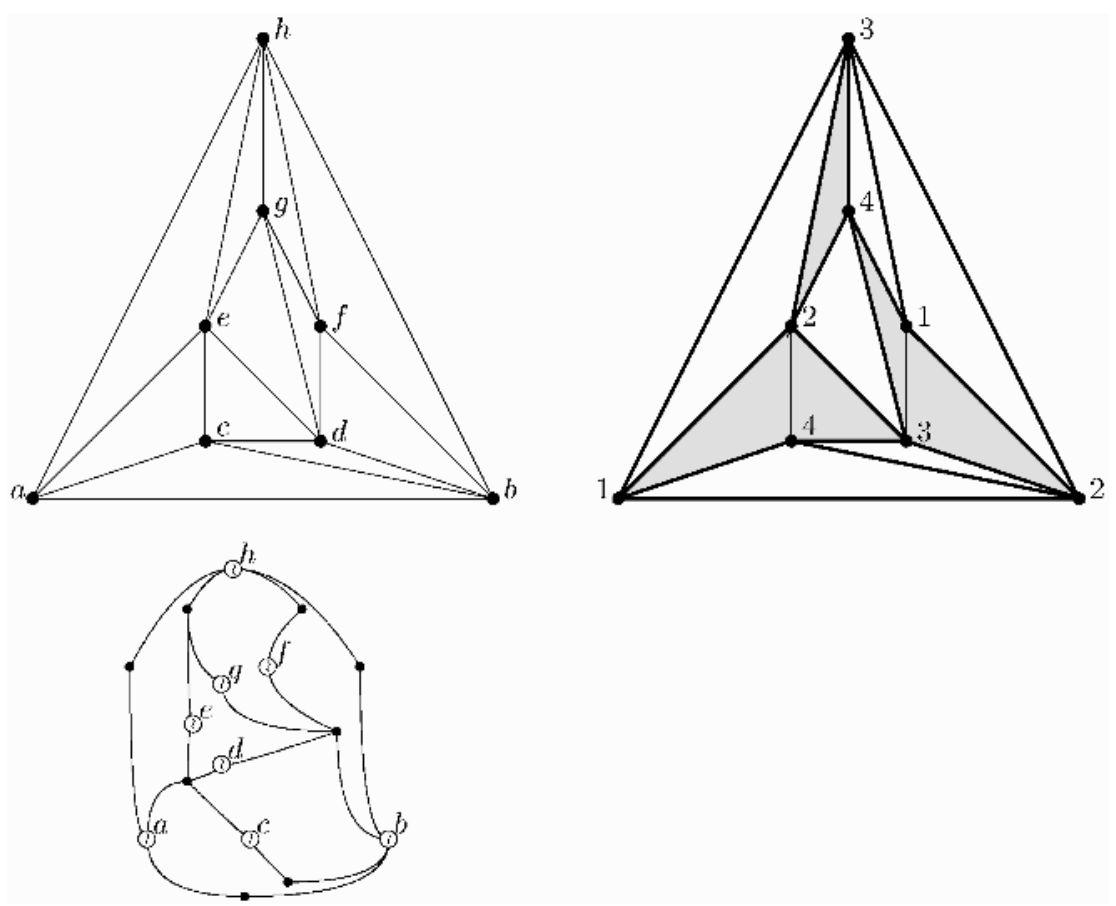

Fig. 1. Using a coloration, a graph $G_{0}$ gives rise to a bipartite plane graph.
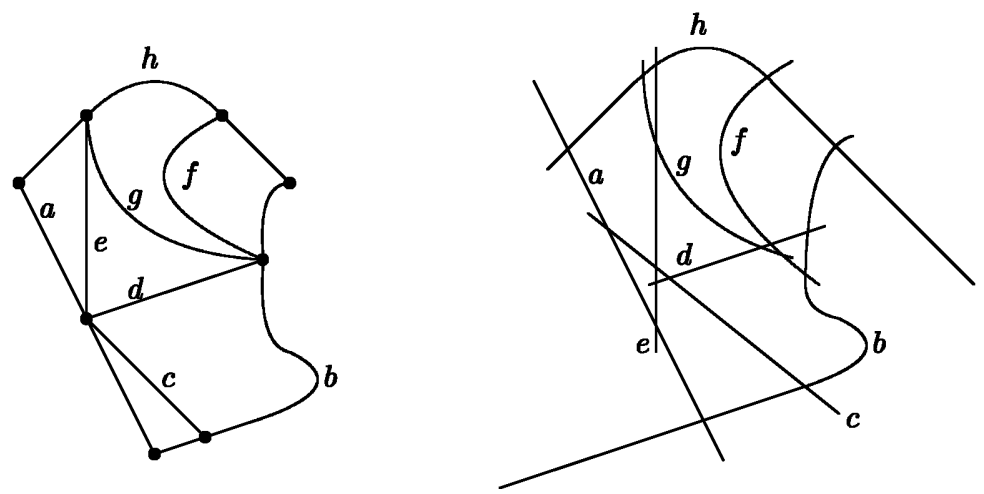

Fig. 2. From the bipartite plane graph shown in Fig 1, we obtain a contact family of pseudo-segments, which by local deformation gives rise to a representation of the graph $G_{0}$ of Fig. 1 as the intersection graph of a family of pseudo-segments.

Using the arc-stretching techniques presented in $[7,8]$, the last two results may be strengthened (see Fig. 3):

Theorem 1. 4-connected 3-colorable plane graphs are contact graphs of a set of segments. 

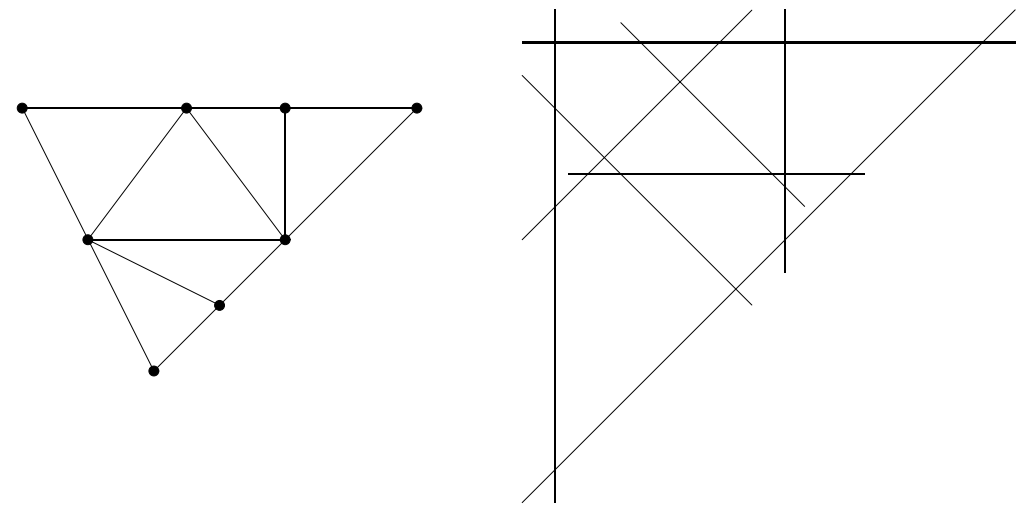

Fig. 3. Using stretching techniques, from the bipartite graph of Fig. 1, we obtain a contact family of segments. By local perturbations, this contact system gives rise to a representation of the graph $G_{0}$ of Fig 1 as the intersection graph of a family of segments (here in 4 directions).

Theorem 2. 4-colored plane graphs without $C_{4}$-separator using 4 colors are intersection graphs of a set of segments.

We shall present a sketch of the proof of these theorems using the following characterization of incidence graphs of a family of segments, which we shall also prove:

Theorem 3. A connected bipartite graph $G=\left(V_{2}, V_{\bullet}, E\right)$ is the incidence graph of a (one-sided) contact family of segments and points if and only if

- G is planar,

- the minimum degree of the vertices in $V_{2}$ is at least 2

$-\forall X \subseteq V$ such that $\left|X \cap V_{2}\right| \geq 2$,

$$
\left|E\left(G_{X}\right)\right| \leq 2\left|X \cap V_{2}\right|+\left|X \cap V_{\bullet}\right|-3
$$
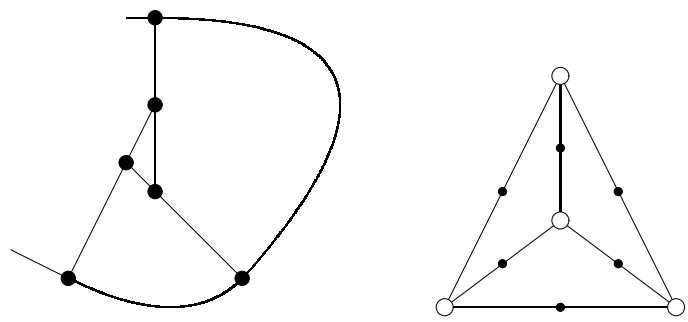

Fig. 4. Representation of $K_{4}$ by a non-stretchable contact family of pseudo-segments. The corresponding incidence graph $\left(V_{2}\right.$ is represented with white vertices, $V \bullet$ with black ones), so that $|E|>2\left|V_{2}\right|+\left|V_{\bullet}\right|-3$. 


\section{Contact Systems of Pseudo-segments and Points}

A finite set of Jordan arcs is called a family of pseudo-segments if every pair of arcs in the set intersects in at most one point. A one-sided contact family of pseudo-segments and points is defined by a couple $(\mathcal{A}, P)$, where:

- $\mathcal{A}$ is a family of pseudo-segments that may touch (on one side only at each contact point) but may not cross, and whose union is connected,

$-P$ is a set of points in the union of the pseudo-segments, including all the extremities of the pseudo-segments.

Such a contact family defines a connected bipartite plane graph $G=\left(V_{2}, V_{\bullet}, E\right)$, its incidence graph, where:

- $V_{2}$ corresponds to the pseudo-segment set,

$-V \bullet$ corresponds to the point set,

- $E$ corresponds to the set of incidences between points and pseudo-segments.

Notice that vertices in $V_{2}$ have minimal degree at least 2.

Moreover, the contact family also defines an orientation of $G$ : if $x \in V$ • corresponds to a point $p$ on a pseudo-segment $S$ corresponding to $y \in V_{2},\{x, y\}$ is oriented from $x$ to $y$ if $p$ is an extremity of $S$ and from $y$ to $x$, otherwise. The orientation thus obtained is such that the indegree of a vertex in $V_{2}$ is exactly 2 and the indegree of a vertex in $V$ • is at most 1 . We call such an orientation a $(2, \leq 1)$-orientation .

The following theorem is quite simple to prove (see [5]):

Theorem 4. A bipartite graph $G=\left(V_{2}, V_{\bullet}, E\right)$ is the incidence graph of a (one-sided) contact family of pseudo-segments and points if and only if

- G is planar,

- $G$ has girth at least 6 ,

- the minimum degree of the vertices in $V_{2}$ is at least 2

$-\forall X \subseteq V$

$$
\left|E\left(G_{X}\right)\right| \leq 2\left|X \cap V_{2}\right|+\left|X \cap V_{\bullet}\right|
$$

In general, representations by contacts of straight line segments raise important difficulties that may be collected into what we call the stretching problem:

Problem 1. When is a contact system of pseudo-segments stretchable, that is: when is it homeomorphic to a contact system of straight line segments?

This problem has been addressed in $[8,7]$, with the following characterization theorem:

Theorem 5. Let $\mathcal{A}$ be a contact system of pseudo-segments. Then, the following conditions are equivalent:

1. $\mathcal{A}$ is stretchable,

2. each subsystem of $\mathcal{A}$ has at least 3 extremal points, unless it has cardinality at most one,

3. $\mathcal{A}$ is extendible. 
where

- An extremal point of a contact system of arcs is a point of the union of the arcs which is interior to no arc.

- A contact system of pseudo-segments is extendible if there exists an arrangement of pseudo-lines such that each pseudo-segment of the contact system is included in a corresponding pseudo-line of the family.

Notice that the equivalence of extendibility and stretchability for contact systems of pseudo-segments is in strong contrast with the difficulty of the decidability problem concerning the stretching of arrangements of pseudo-lines (this problem is NP-hard, as proved by Mnëv [19,20]; see also Shor [26] and RichterGebert [22]).

\section{Deficiency and $(2, \leq 1)$-Orientation}

In order to make use of Theorem 5 to characterize those bipartite graphs that are representable by a contact family of segments, we first need to prove an orientation theorem. For that, we need few definitions and lemmas.

In the following, we consider a connected bipartite graph $G=\left(V_{\imath}, V_{\bullet}, E\right)$. Let $V=V_{2} \cup V_{\bullet}$. Given a subset $A \subseteq V$, we introduce the notation $A_{2}=A \cap V_{2}$ and $A_{\bullet}=A \cap V_{\bullet}$. By extension, if $\mathrm{f}(x)$ is a subset of $V$, we employ the notation $\mathrm{f}_{2}(x)=\mathrm{f}(x) \cap V_{2}$ and $\mathrm{f}_{\bullet}(x)=\mathrm{f}(x) \cap V_{\bullet}$. We denote by $\mathcal{N}$ the neighborhood function defined by $\mathcal{N}(X)=X \cup\{y \in V, \exists x \in X:\{x, y\} \in E\}$. Observe that $X \subseteq \mathcal{N}(X)$.

\subsection{Deficiency}

\section{Definition 1.}

the deficiency $\rho$ of a subset $X \subseteq V$ is

$$
\begin{aligned}
\rho(X) & =2\left|X_{2}\right|+\left|X_{\bullet}\right|-\left|E\left(G_{X}\right)\right| \\
\rho_{\min }(X) & =\min _{X \subseteq Y} \rho(Y) \\
\operatorname{Clos}(X) & =\bigcup_{\substack{X \subseteq Y \\
\rho(Y)=\rho_{\min }(X)}} Y
\end{aligned}
$$$$
\text { the minimal deficiency } \rho_{\min } \text { of } X \text { is }
$$$$
\text { the deficiency closure Clos of } X \text { is }
$$

Lemma 1. The function $\rho$ is semimodular, that is, $\forall X_{1}, X_{2} \subseteq V$ :

$$
\rho\left(X_{1} \cup X_{2}\right)+\rho\left(X_{1} \cap X_{2}\right) \leq \rho\left(X_{1}\right)+\rho\left(X_{2}\right)
$$

Proof. This is a direct consequence of the inequality

$$
\left|E\left(G_{X_{1} \cup X_{2}}\right)\right| \geq\left|E\left(G_{X_{1}}\right)\right|+\left|E\left(G_{X_{2}}\right)\right|
$$

Lemma 2. Let $X \subseteq V$. Then $\rho(\operatorname{Clos}(X))=\rho_{\min }(X)$. 
Proof. Assume $X_{1}, X_{2}$ are subsets of $V$ containing $X$ such that $\rho\left(X_{1}\right)=\rho\left(X_{2}\right)=$ $\rho_{\min }(X)$. Then, as $X \subseteq X_{1} \cap X_{2}$, we get $\rho\left(X_{1} \cap X_{2}\right) \geq \rho_{\min }(X)$ and, according to (3), $\rho\left(X_{1} \cup X_{2}\right) \leq \rho_{\min }(X)$. Thus $\rho\left(X_{1} \cup X_{2}\right)=\rho_{\min }(X)$. By induction we deduce that $\rho(\operatorname{Clos}(X))=\rho_{\min }(X)$.

Lemma 3. For any $A \subseteq V_{2}$,

$$
\mathcal{N}(A) \subseteq \mathcal{N}\left(\operatorname{Clos}_{2}(A)\right)=\operatorname{Clos}(A)
$$

Proof. For any $X \in V$, we have $\rho\left(\mathcal{N}\left(X_{2}\right)\right) \leq \rho(X)$, as the addition to $X$ of a vertex in $V \bullet$ having at least one neighbor in $X$ doesn't increase $\rho(X)$ and as the deletion of a vertex in $V$ • having no neighbor in $X$ decreases $\rho(X)$ by 1 . Hence $\rho\left(\mathcal{N}\left(X_{2}\right)\right) \leq \rho(X)$ and equality may only occur if $X \subseteq \mathcal{N}\left(X_{2}\right)$.

According to this property, as $\rho\left(\mathcal{N}\left(Y_{2}\right)\right) \leq \rho(Y)$ and as equality implies $Y \subseteq \mathcal{N}\left(Y_{2}\right)$, we have:

$$
\operatorname{Clos}(A)=\bigcup_{\substack{A \subseteq Y \\ \rho \subseteq \rho_{\min }(A)}} Y=\bigcup_{\substack{A \subseteq Y \\ \rho(Y)=\rho_{\min }(A)}} \mathcal{N}\left(Y_{2}\right)=\mathcal{N}\left(\bigcup_{\substack{A \subseteq Y \\ \rho(Y)=\rho_{\min }(A)}} Y_{2}\right)=\mathcal{N}\left(\mathrm{Clos}_{2}(A)\right)
$$

Moreover, as $A \subseteq \operatorname{Clos}_{\imath}(A), \mathcal{N}(A) \subseteq \mathcal{N}\left(\operatorname{Clos}_{\imath}(A)\right)$.

\section{$3.2(2, \leq 1)$-Orientation}

Definition 2. $A(2, \leq 1)$-orientation $\mathcal{O}$ of a bipartite graph $G$ is an orientation such that each vertex in $V_{2}$ has indegree exactly 2 and every vertex in $V$ • has indegree at most 1 . A source of the $(2, \leq 1)$-orientation is a vertex with null indegree. Given a subset $X \subseteq V$, a vertex $x \in X$ is a relative source of $X$ for $\mathcal{O}$ if it has a null indegree in $G_{X}$. We note $\operatorname{Source}(\mathcal{O}, X)$ the set of the relative sources of $X$ for $\mathcal{O}$.

The two following lemmas justify the term of deficiency for $\rho$.

Lemma 4 ([5]). A connected bipartite graph $G$ has a $(2, \leq 1)$-orientation if and only the minimal degree of vertices in $V_{2}$ is at least 2 and if

$$
\forall X \subseteq V,\left|E\left(G_{X}\right)\right| \leq 2\left|X_{2}\right|+\left|X_{\bullet}\right|
$$

Lemma 5. Let $G$ be a bipartite planar graph and a $(2, \leq 1)$-orientation of $G$. Let $X \subseteq V$. Then $\rho(X)$ is equal to the sum of the number of sources of $G$ in $X$ and of the number of arcs entering $X$ from $V \backslash X$.

Proof. The result is easily obtained by summing up the indegrees of the vertices in $X$.

Lemma 6. Let $\mathcal{O}$ be a $(2, \leq 1)$-orientation of $G$ and let $X \subseteq V$. Then there exists a $(2, \leq 1)$-orientation $\mathcal{O}^{\prime}$ of $G$ such that

$$
\operatorname{Source}(\mathcal{O}, \operatorname{Clos}(X)) \subseteq \operatorname{Source}\left(\mathcal{O}^{\prime}, \operatorname{Clos}(X)\right) \subseteq \operatorname{Source}\left(\mathcal{O}^{\prime}, V\right)
$$


Proof. Let $Y$ be the subset of $V \backslash \operatorname{Clos}(X)$ formed by the vertices $y$ such that there exists a directed path from $y$ to a vertex $x \in \operatorname{Clos}(X)$. We proceed iteratively, while decreasing the number of sources of $G$ in $Y$.

If $Y$ includes no source of $\mathcal{O}$, then any vertex in $Y$ has its incoming edges incident to vertices in $Y \cup \operatorname{Clos}(X)$. Thus $\rho(\operatorname{Clos}(X) \cup Y) \leq \rho(\operatorname{Clos}(X))=\rho_{\min }(X)$, a contradiction. Hence $Y$ is empty, thus $\operatorname{Source}\left(\mathcal{O}^{\prime}, \operatorname{Clos}(X)\right) \subseteq \operatorname{Source}\left(\mathcal{O}^{\prime}, V\right)$.

Otherwise, let $y$ be source of $G$ in $Y$. By assumption, there exists a directed path from $y$ to a vertex $x \in X$. According to Lemma 3, $\operatorname{Clos}(X)=\mathcal{N}\left(\operatorname{Clos}_{\imath}(X)\right)$. Thus if the directed path has minimal length, $x \in \operatorname{Clos} \bullet(X)$. Reorienting the directed path from $x$ to $y$ decreases the number of sources in $Y$, decreases $Y$ and gives rise to a new $(2, \leq 1)$-orientation of $G$. As the reorientation may not have killed a relative source of $\operatorname{Clos}(X)$, we have $\operatorname{Source}(\mathcal{O}, \operatorname{Clos}(X)) \subseteq$ $\operatorname{Source}\left(\mathcal{O}^{\prime}, \operatorname{Clos}(X)\right)$.

Lemma 7. Let $\mathcal{O}$ be a $(2, \leq 1)$-orientation of $G$ and let $X \subseteq V$. Assume that any vertex $x \in X_{2}$ which has a neighbor out of $X$ has at least two neighbors in $X$ and that $\operatorname{Source}(\mathcal{O}, \operatorname{Clos}(X)) \subseteq \operatorname{Source}(\mathcal{O}, V)$. Then there exists a $(2, \leq 1)$-orientation $\mathcal{O}^{\prime}$ of $G$, which differs with $\mathcal{O}$ only on $E(\operatorname{Clos}(X))$, such that:

$$
\operatorname{Source}(\mathcal{O}, \operatorname{Clos}(X)) \cap X \subseteq \operatorname{Source}\left(\mathcal{O}^{\prime}, \operatorname{Clos}(X)\right) \subseteq \operatorname{Source}\left(\mathcal{O}^{\prime}, V\right) \cap X
$$

Proof. We proceed by induction on the cardinality of $\operatorname{Source}(\mathcal{O}, \operatorname{Clos}(X)) \backslash X$.

If $\operatorname{Source}(\mathcal{O}, \operatorname{Clos}(X)) \subseteq X$, we are done. Otherwise, let $s \in \operatorname{Source}(\mathcal{O}$, $\operatorname{Clos}(X)) \backslash X$. Let $Y$ be the subset of the vertices $x \in \operatorname{Clos}(X)$ reachable from $s$ by a directed path. If $Y \cap X=\emptyset$, then $\rho(\operatorname{Clos}(X) \backslash Y)<\rho(\operatorname{Clos}(X))$ although $X \subseteq \operatorname{Clos}(X) \backslash Y$, contradicting the minimality of $\rho(\operatorname{Clos}(X))$. Thus $Y \cap X \neq \emptyset$. Let $v_{1}=s, \ldots, v_{k}$ be a minimal length directed path from $s$ to a vertex in $X$. If $v_{k} \in X_{2}$, there exists an outgoing arc at $v_{k}$ to a vertex $v_{k+1} \in X_{\bullet}$, as $v_{k}$ has degree at least 2 in $X$ by assumption, as the indegree of $v_{k}$ is 2 and as $v_{k}$ has an incoming edge from $v_{k-1} \notin X$. Reversing the orientation of the path $v_{1}, \ldots, v_{k}\left(\operatorname{resp} . v_{1}, \ldots, v_{k+1}\right)$ if $v_{k} \in X \bullet\left(\right.$ resp. $\left.v_{k} \in X_{\imath}\right)$, we obtain a new $(2, \leq 1)$-orientation $\mathcal{O}^{\prime}$ of $G$. As this orientation differs with $\mathcal{O}$ on $G_{\operatorname{Clos}(X)}$ only, we still have $\operatorname{Source}\left(\mathcal{O}^{\prime}, \operatorname{Clos}(X)\right) \subseteq \operatorname{Source}\left(\mathcal{O}^{\prime}, V\right)$. As we may not have killed a source in $X$, Source $(\mathcal{O}, \operatorname{Clos}(X)) \cap X \subseteq \operatorname{Source}\left(\mathcal{O}^{\prime}, \operatorname{Clos}(X)\right)$. Moreover, $|\operatorname{Source}(\mathcal{O}, \operatorname{Clos}(X)) \backslash X|$ decreased by one.

Definition 3. A subset $X$ of vertices of a connected plane graph $G$ is a disk if any vertex of $X$ having a neighbor out of $X$ belongs to the outer face of $G_{X}$ and $X=\mathcal{N}\left(X_{2}\right)$.

Theorem 6. Assume $G=\left(V_{\imath}, V_{\bullet}, E\right)$ is a connected bipartite plane graph such that the minimum degree of vertices in $V_{2}$ is at least 2 and such that $\forall X \subseteq V$,

$$
\left|X_{\imath}\right| \geq 2 \Longrightarrow\left|E\left(G_{X}\right)\right| \leq 2\left|X_{\imath}\right|+\left|X_{\bullet}\right|-3
$$

Then $G$ has a $(2, \leq 1)$-orientation $\mathcal{O}$ such that, for any disk $X$ with $\left|X_{2}\right| \geq 2$, we have

$$
|\operatorname{Source}(\mathcal{O}, X) \cap \operatorname{Extr}(X)| \geq 3
$$

where $\operatorname{Extr}(X)$ denotes the vertex set of the outer face of $G_{X}$. 
Proof. According to Lemma 4, $G$ has a $(2, \leq 1)$-orientation $\mathcal{O}_{0}$.

We prove by induction over $(|V|,|V \backslash \operatorname{Extr}(V)|)$ that the required $(2, \leq 1)$ orientation $\mathcal{O}$ may be found, with the additional properties that the sources of $\mathcal{O}_{0}$ in $\operatorname{Extr}(V)$ are also sources of $\mathcal{O}$.

Let $A=\operatorname{Extr}(V)$. According to Lemmas 6 and 7 , there exists a $(2, \leq 1)$ orientation $\mathcal{O}_{1}$ of $G$, such that

$$
\operatorname{Source}\left(\mathcal{O}_{0}, V\right) \cap A \subseteq \operatorname{Source}\left(\mathcal{O}_{1}, \operatorname{Clos}(A)\right) \subseteq \operatorname{Source}\left(\mathcal{O}_{1}, V\right) \cap A
$$

Let $B_{1}=V \backslash \operatorname{Clos}(A), B_{2}=\mathcal{N}\left(B_{1}\right) \backslash B_{1}$ and $B_{3}=\mathcal{N}\left(B_{2}\right) \backslash B_{2}$. According to Lemma $3, B_{2} \subseteq V_{\bullet}$ and $B_{3} \subseteq V_{2}$. Moreover, $B_{3} \subseteq \operatorname{Extr}\left(B_{1} \cup B_{2} \cup B_{3}\right)$. Let $G^{\prime}$ be the directed bipartite plane graph obtained from $G$ as follows: First, we remove all the vertices not in $B_{1} \cup B_{2} \cup B_{3}$ and all the arcs oriented from $B_{2}$ to $B_{3}$. Finally, for every $v \in B_{3}$, we add two new vertices $v_{1}$ and $v_{2}$ on the outer face with $\operatorname{arcs}\left(v_{1}, v\right)$ and $\left(v_{2}, v\right)$. Let $C$ be the corresponding set of added black vertices. Then, $B_{3} \cup C$ belong to the outer face of $G^{\prime}$ and the orientation of $G^{\prime}$ is a $(2, \leq 1)$-orientation having every vertex in $C$ as a source. As $V_{2}\left(G^{\prime}\right)$ is either strictly included in $V_{2}(G)$ or is equal but then $\left|\operatorname{Extr}_{2}\left(V\left(G^{\prime}\right)\right)\right|$ is strictly greater than $\left|\operatorname{Extr}_{2}(V(G))\right|$, the induction applies. Thus there exists a $(2, \leq 1)$ orientation $\mathcal{O}_{2}$ of $G^{\prime}$, such that any vertex in $C$ is a source of $\mathcal{O}_{2}$ and such that (6) holds for any disk $X$ with $\left|X_{2}\right| \geq 2$.

Let $\mathcal{O}$ be the orientation of $G$ induced by $\mathcal{O}_{1}$ on $G_{V \backslash B_{1}}$ and $\mathcal{O}_{2}$ on $G_{B_{1} \cup B_{2}}$. By construction, $\mathcal{O}$ is a $(2, \leq 1)$-orientation of $G$ such that $\operatorname{Source}\left(\mathcal{O}_{0}, V\right) \cap \operatorname{Extr}(V) \subseteq$ $\operatorname{Source}(\mathcal{O}, V)$ and $\operatorname{Source}(O, \operatorname{Clos}(A)) \subseteq \operatorname{Source}(O, V) \cap \operatorname{Extr}(V)$.

Let $X$ be a disk of $G$ such that $\left|X_{l}\right| \geq 2$ and let $Y=X \cap \operatorname{Clos}(A)$.

Assume $\left|Y_{2}\right| \geq 2$. According to (5), we get $\left|E\left(G_{Y}\right)\right| \leq 2\left|Y_{2}\right|+\left|Y_{\bullet}\right|-3$. According to Lemma 5, $|\operatorname{Source}(O, Y)| \geq 3$. Moreover, as $X$ is a disk and as $\operatorname{Clos}(A)$ has no entering arc, every relative source of $Y$ belongs to the outer face of $G_{X}$. Thus $\mid$ Source $(O, X) \cap \operatorname{Extr}(X)|\geq| \operatorname{Source}(O, Y) \cap \operatorname{Extr}(X) \mid \geq 3$.

Otherwise, $X_{2}$ is included in the vertex set of $G^{\prime}$. In $G^{\prime}$ we thus get

$$
\left|\operatorname{Source}\left(O_{2}, X\right) \cap \operatorname{Extr}(X)\right| \geq 3 \text {. }
$$

By construction of $G^{\prime}$ and $\mathcal{O}$, we deduce $|\operatorname{Source}(O, X) \cap \operatorname{Extr}(X)| \geq 3$.

Theorem 7. A connected bipartite graph $G=\left(V_{2}, V_{\bullet}, E\right)$ is the incidence graph of a (one-sided) contact family of segments and points if and only if

- $G$ is planar,

- the minimum degree of the vertices in $V_{2}$ is at least 2

$-\forall X \subseteq V$ such that $\left|X \cap V_{2}\right| \geq 2$,

$$
\left|E\left(G_{X}\right)\right| \leq 2\left|X \cap V_{2}\right|+\left|X \cap V_{\bullet}\right|-3
$$

Proof. According to Theorem 4, $G$ is the contact graph of a family of pseudosegments $\mathcal{A}$. According to Theorem $6, G$ has a $(2, \leq 1)$-orientation $\mathcal{O}$, such that any disk $X$ with $\left|X_{2}\right| \geq 2$ has at least 3 relative sources on the outer face of $G_{X}$. Thus, according to Theorem $5, \mathcal{A}$ is stretchable into a contact family of segments. 
Corollary 1. A graph $G=(V, E)$ is the intersection graph of a (one-sided) simple contact family of segments if and only if

- $G$ is planar,

- any subgraph $H \subseteq G$ of order $n_{H} \geq 2$ has its size bounded by: $m_{H} \leq 2 n_{H}-3$.

Proof. Apply Theorem 7 to the bipartite graph obtained by subdividing each edge of $G$ exactly once.

In [2], it is proved that any 4-connected 3-colorable plane graphs is the contact graphs of a set of pseudo-segments. It is also proved in [2] that the assumptions of Theorem 6 hold for the incidence graph of the contact system. Thus, we get:

Corollary 2. Any 4-connected 3-colorable plane graphs is the contact graphs of a family of segments.

In [2], representations of planar graphs by intersection of pseudo-segments are obtained using local perturbations of contact systems of pseudo-segments. The assumptions of Theorem 6 are proved to hold for the contact system in [2]. Thus, using Theorem 7 and a perturbation argument, this theorem may be strengthened:

Corollary 3. Any 4-colored planar graph without induced $C_{4}$ using 4 colors is the intersection graph of a family of straight line segments.

\section{Open Problems}

It is not difficult to prove that any contact family of pseudo-segments is homeomorphic to a contact family of polylines composed by three segments.

Problem 2. Is any contact family of pseudo-segments homeomorphic to a contact family of polylines composed by two segments?

It is known that every planar graph is representable as the contact graph of a family of triangles[10]. Using stretching techniques, this result might extend:

Problem 3. Is any planar linear hypergraph representable as the contact hypergraph of a family of triangles?

Scheinerman's conjecture may be straightened as follows, as a self-dual statement:

Problem 4. Is any planar linear hypergraph representable as the intersection hypergraph of a family of segments?

As the coloration seems to play a central role, we may also ask:

Problem 5. Is any planar graph $G$ representable as the intersection graph of a family of segments in $\chi(G)$ directions? 


\section{References}

1. J. Czyziwicz, E. Kranakis, and J. Urrutia, A simple proof of the representation of bipartite planar graphs as the contact graphs of orthogonal straight line segments, Information Processing Letters (1998), no. 66, 125-126.

2. N. de Castro, F. J. Cobos, J.C. Dana, and A. Márquez, Triangle-free planar graphs as segment intersection graphs, Journal of Graph Algorithms and Applications 6 (2002), no. 1, 7-26.

3. H. de Fraysseix, Local complementation and interlacement graphs, Discrete Mathematics 33 (1981), 29-35.

4. $ـ$ A Characterization of Circle Graphs, European Journal of Combinatorics 5 (1984), 223-238.

5. H. de Fraysseix and P. Ossona de Mendez, Intersection Graphs of Jordan Arcs, Contemporary Trends in Discrete Mathematics, DIMACS Series in Discrete Mathematics and Theoretical Computer Science, vol. 49, DIMATIA-DIMACS, 1999, Štiřin 1997 Proc., pp. 11-28.

6. _ On a Characterization of Gauss Codes, Discrete and Computational Geometry 2 (1999), no. 2.

7., Barycentric systems and stretchability, Discrete Applied Mathematics (2004), (submited).

8. _ Stretching of Jordan arc contact systems, Graph Drawing, Lecture Notes in Computer Science, vol. 2912, Springer, 2004, pp. 71-85.

9. H. de Fraysseix, P. Ossona de Mendez, and J. Pach, Representation of planar graphs by segments, Intuitive Geometry 63 (1991), 109-117.

10. H. de Fraysseix, P. Ossona de Mendez, and P. Rosenstiehl, On triangle contact graphs, Combinatorics, Probability and Computing 3 (1994), 233-246.

11. G. Ehrlich, S. Even, and R.E. Tarjan, Intersection graphs of curves in the plane, Journal of Combinatorial Theory 21(B) (1986), 8-20.

12. C.F. Gauss, Werke, pp. 272 and 282-285, Teubner Leipzig, 1900.

13. I.B.-A. Hartman, I. Newman, and R. Ziv, On grid intersection graphs, Discrete Math. 87 (1991), 41-52.

14. Petr Hliněny, Contact graphs of line segments are NP-complete, Discrete Mathematics 235 (2001), no. 1-3, 95-106.

15. J. Kratochvíl, String graphs I : the number of critical nonstring graphs is infinite, J. Combin. Theory Ser. B 52 (1991), 53-66.

16. _ String graphs II : Recognizing string graphs is NP-hard, J. Combin. Theory Ser. B 52 (1991b).

17. J. Kratochvíl and J. Matoušek, String graphs requiring exponential representations, J. Combin. Theory Ser. B 52 (1991), 1-4.

18. Intersection graphs of segments, Journal of Combinatorial Theory 62(B) (1994), no. 2, 289-315.

19. N.E. Mnëv, On manifolds of combinatorial types of projective configurations and convex polyhedra, Soviet Math. Deokl. (1985), no. 32, 335-337.

20. - The universality theorems on the classification problem of configuration varieties and convex polytopes varieties, Topology and Geometry - Rohlin Seminar (Berlin) (O. Ya. Viro, ed.), Lecture Notes in Mathematics, vol. 1346, SpringerVerlag, 1988, pp. 527-544.

21. J. Pach and G. Tóth, Which crossing number is it anyway?, Journal of Combinatorial Theory, Ser. B 80 (2000), no. 2, 225-246. 
22. J. Richter-Gebert, Realization spaces of polytopes, Lecture Notes in Mathematics 1643 (1996).

23. P. Rosenstiehl, A new proof of the Gauss interlace conjecture, Advances in Applied Mathematics 23 (1999), no. 1, 3-13.

24. M. Schaefer, E. Sedgwick, and D. Stefankovič, Recognizing string graphs in NP, Journal of Computer and System Sciences 67 (2003), no. 2, 365-380.

25. E.R. Scheinerman, Intersection classes and multiple intersection parameters of graphs, Ph.D. thesis, Princeton University, 1984.

26. P. W. Shor, Stretchability of pseudolines is NP-hard, DIMACS Series in Discrete Mathematics and Theoretical Computer Science 4 (1991), 531-554.

27. F.W. Sinden, Topology of thin RC-circuits, Bell System Tech. J. (1966), 1639-1662.

28. D. West, Opem problems, SIAM J. Discrete Math. Newslett. 2 (1999), no. 1, 10-12. 